\title{
QUARTZ CRYSTAL OSCILLATOR CLASSIFICATION BY DIPOLAR ANALYSIS
}

\author{
R. Brendel ${ }^{1}$, F. Chirouf ${ }^{1}$, D. Gillet ${ }^{1}$, N. Ratier ${ }^{1}$, F. Lardet-Vieudrin ${ }^{1}$, M. Addouche $^{2}$, J. Delporte $^{3}$ \\ ${ }^{1}$ Laboratoire de Physique et Métrologie des Oscillateurs du CNRS associé à l'Université de Franche-Comté \\ 32, Avenue de l'Observatoire - 25044 Besançon Cedex - France \\ ${ }^{2}$ Laboratoire d'Astrophysique de l'Observatoire de Besançon \\ 41, Avenue de l'Observatoire - 25030 Besançon Cedex - France \\ ${ }^{3}$ Centre National d'Études Spatiales \\ 18, Avenue E. Belin - 31055 Toulouse Cedex - France
}

\begin{abstract}
The dipolar method associated with a nonlinear time domain simulation program make up a powerful tool to analyze high Q-factor circuits like quartz crystal oscillators. After a brief remainder of the dipolar method, the paper will attempt to identify the main amplifier characteristics such as limitation mechanism, input and output impedances, etc. and to point out their influence on the amplifier dipolar impedance. The effect of the amplifier nonlinearities on the oscillator characteristics, as well as the particular role of the crystal parallel capacitance are particularly emphasized.
\end{abstract}

Keywords - Oscillators, modeling, dipolar method

\section{INTRODUCTION}

The dipolar analysis is a non-linear time domain method well suited to describe the behavior of high Q-factor crystal oscillators [1-3]. In this method, to overcome the unacceptably long transient time needed to reach the steady state of high-Q circuits, the oscillator amplifier is separated from the resonator and replaced by a sinusoidal current source. The amplifier then behaves like a dipole the impedance of which is evaluated at the resonator frequency. An electrical simulation program like SPICE is used to perform a set of transient analyses of higher and higher amplitude so as to obtain the variation of both the dipolar resistance and reactance as a function of the current source amplitude.

When such a dipole is connected to a crystal resonator, oscillation occurs when the non-linear dipolar impedance is equal and opposite to the resonator impedance [4-5]. This leads to a non-linear equation that can be used to obtain the oscillation start-up condition, the steady state current amplitude and oscillation frequency or a time domain non-linear differential equation whose solution is the oscillation loop current. Because of the high resonator Q-factor, the oscillation loop current is almost perfectly sinusoidal so that asymptotic method, like slowly varying functions method [6-7] can be used to obtain both amplitude and frequency transients without having to solve the initial nonlinear differential equation. The effect of noise can also be calculated by using a perturbation method in the vicinity of the steady state [8], and for noise Fourier components close to the carrier, perturbation results in both amplitude and frequency modulation from which amplitude and phase noise spectra are easily calculated.
An implementation of the dipolar method achieved in the ADOQ program ("Analyse Dipolaire des Oscillateurs à Quartz") is able to compute the steady state features of the oscillator: oscillation frequency, amplitude, drive level, signal shape and gives the start-up condition for oscillation to occur [2]. Also the program performs accurate oscillator sensitivity calculation to various parameters (component value, supply voltage, etc.) as well as amplitude and phase noise spectra [8].

\section{SimUlation PRINCIPLE}

The nonlinear dipolar method consists in representing an oscillator by the quartz motional branch connected across a non-linear dipole amplifier (Fig. 1). The parallel capacitance and, if needed, the pulling capacitance, are included in the amplifier dipole. The impedance of the nonlinear dipole amplifier strongly depends on the current amplitude and weakly depends on the current frequency. It can be represented by a nonlinear resistance in series with a nonlinear reactance, symbolized for convenience in Fig. 1 by an inductance, that vary with the amplitude of the current.

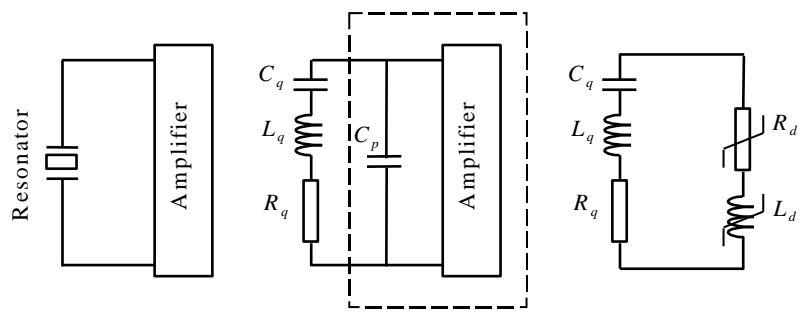

Fig. 1. Dipolar representation of a quartz crystal oscillator.

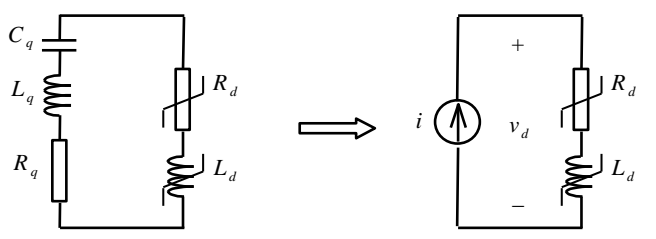

Fig. 2. Dipolar analysis of the oscillator amplifier.

Because of the resonator's high quality factor, the loop current in the oscillator is almost perfectly sinusoidal. Thus, the nonlinear behavior of the dipolar amplifier can be ob- 
tained by replacing the resonator motional branch by a sinusoidal current source, and performing a set of transient analyses with increasing amplitudes by using an electrical simulator like SPICE (Fig. 2).

The complex impedance of the nonlinear dipole amplifier is obtained, for each current amplitude value by performing a Fourier analysis on the steady state voltage across the dipole. Nonlinear amplifier resistance and reactance are obtained by giving the sinusoidal current a larger and larger amplitude (Fig. 3).
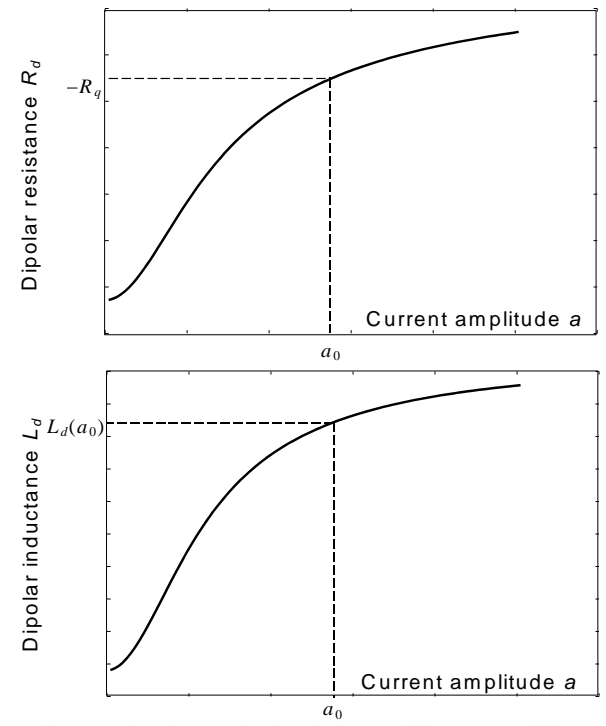

Fig. 3. Dipolar analysis of the oscillator amplifier.

The nonlinear differential equation of the oscillator is given by (1), where $\omega_{q}$ is the quartz series resonant frequency and $a$ is the amplitude of the current fundamental component $i(t)$. In quartz crystal oscillator $L_{q}>L_{d}$ so that:

$$
\begin{gathered}
\frac{d^{2} i}{d t^{2}}+\frac{1}{L_{q}}\left(R_{q}+R_{d}(a)\right) \frac{d i}{d t}+\omega_{q}^{2}\left(1-\frac{L_{d}(a)}{L_{q}}\right) i=0 \\
\omega_{q}^{2}=\frac{1}{L_{q} C_{q}}
\end{gathered}
$$

The solution of this equation is taken under the form shown in (3) where $a(t)$ and $\varphi(t)$ are slowly varying functions of time.

$$
i=a(t) \cos \left(\omega_{q} t+\varphi(t)\right)
$$

At low current amplitude level, the damping term of (1) should have a negative value to insure increasing amplitude solution. If $R_{d s}$ is the value of the nonlinear dipolar resistance at very low current amplitude, the start-up condition takes the form (4).

$$
R_{q}+R_{d s}<0
$$

As the oscillation amplitude increases, the dipolar resistance increases so that the value of the damping term increases. The steady state amplitude $a_{0}$ is reached when this term becomes zero as given by (5) and Fig. 3 .

$$
R_{q}+R_{d}\left(a_{0}\right)=0
$$

$$
\omega_{0}^{2}=\omega_{q}^{2}\left(1-\frac{L_{d}\left(a_{0}\right)}{L_{q}}\right)
$$

The steady state frequency is then given by (6) where $L_{d}\left(a_{0}\right)$ is the value of the steady state dipolar inductance deduced from the curve in Fig. 3.

\section{OSCILLATOR AMPLIFIER CLASSIFICATIONS}

A large number of amplifier circuits can be used to build an oscillator each having its own advantages and drawbacks. The choice among the different circuits is of course dictated by the application the oscillator will be used in, and also by technical considerations such as: frequency range, output power range, frequency stability, output waveform, phase noise, etc. [9]. Even for a given set of specifications there are many different circuits able to meet them and a particular choice is often a matter of personal or collective experience or skill rather than the conclusion of a methodical analysis. It is not our claim to give a methodical reasoning leading to an optimal circuit, but more modestly, to give the designer an efficient tool to help him to make a good choice.

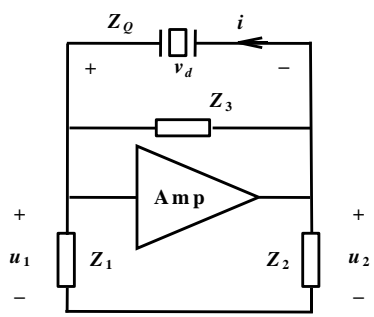

Fig. 4. Amplifier representation.

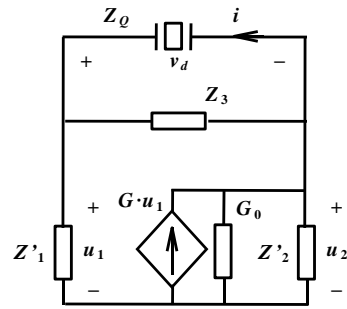

(a)

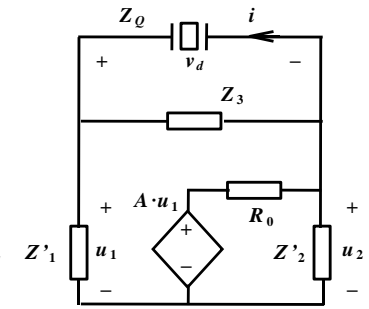

(b)
Fig. 5. Controlled current or voltage source representation of the amplifier with current (a) or voltage (b) controlled source.

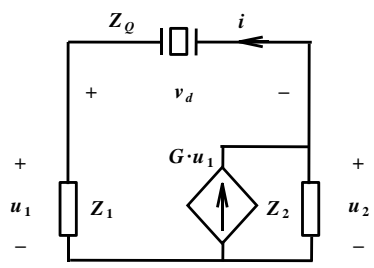

Fig. 6. Reduced amplifier representation.

From the dipolar point of view, the amplifiers used in the oscillator circuits can be classified according several ways described in the following sections. So as to explain the classification method, the black box amplifier shown in Fig. 1 will be taken under the form shown in Fig. 4 where $Z_{1}$ and $Z_{2}$ are the amplifier input and output impedances in pa- 
rallel with possible external impedances, while $Z_{3}$ mainly represents the effect of the resonator's parallel capacitance considered as a part of the amplifier circuit as stated in section II in parallel with a possible biasing impedance. Although it is possible, in a linear small signal analysis, to include $Z_{3}$ into $Z_{1}$ and $Z_{2}$ by a simple circuit transformation, in some case it is wiser to keep it as a separate impedance because of the particular role it plays in the dipolar impedance as it will be shown later.

The amplifier itself can be represented either by a voltage controlled current source (Fig. 5.a) or by an equivalent voltage controlled voltage source (Fig. 5.b). In the linear case, by a simple circuit transformation, either one of the two representations in Fig. 5 can be reduced to the form shown in Fig. 6. Note that in Figs. 4, 5, and 6, the voltage reference node is not necessarily the ground node.

A general expression of the small signal dipolar impedance is obtained by replacing the resonator in Fig. 6 by a current source of same frequency as shown in Fig. 7.

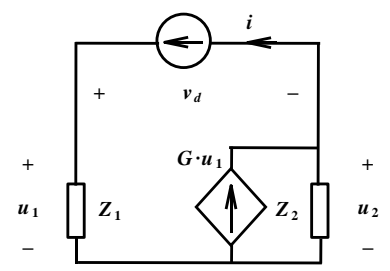

Fig. 7. Dipolar impedance characterization.

By expressing the dipolar voltage $v_{d}$ as a function of the current $i$, it is simple to obtain the small signal dipolar impedance under the form (7). From the dipolar point of view, the transconductance $G$ (or the voltage gain $A$ ) can be real or complex, linear or nonlinear moreover all variables of the right hand side in (7) might be function of the current amplitude $a$, so that the nonlinear dipolar impedance take the form (8).

$$
\begin{aligned}
Z_{d s} & =Z_{1}+Z_{2}-G Z_{1} Z_{2} \\
Z_{d} & =R_{d}(a)+j X_{d}(a)
\end{aligned}
$$

\section{AMPLITUDE LIMITATION}

It is well known that the oscillation amplitude is determined by the nonlinear behavior of the amplifier. From this point of view, the amplifier circuits can be split into several categories such as: saturation or cutoff limitation, hard or soft limitation, symmetrical or non-symmetrical limitation, with possible combination of these various limitation mechanisms.

\section{A. Hard Saturation Limiting}

In the simple behavioral oscillator circuit shown Fig. 8.a, the amplifier is an ideal circuit having a real input impedance $R$, and a linear real positive gain $A$ limited by symmetrical saturation as shown in Fig. 8.b. In this case, because the amplifier is assumed ideal with a zero output impedance, it cannot be reduced to a transconductance amplifier.
The nonlinear transfer function of the amplifier is determined by the conditions (9).

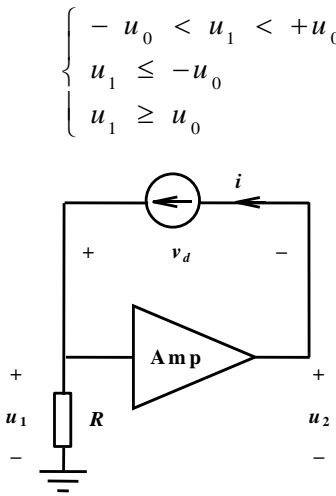

(a)

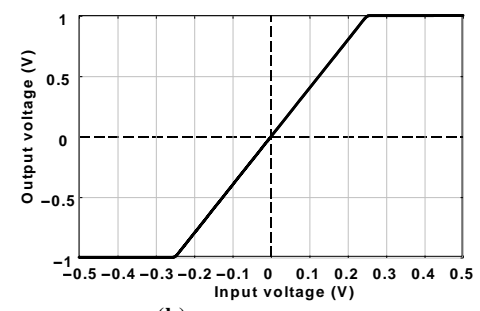

(b)
Fig. 8. Simple behavioral oscillator with saturation limiting. Values used are: $A=4, R=100 \Omega, V_{\text {sat }}=1 \mathrm{~V}$.

For small value of the current $i$, the dipolar impedance $Z_{d s}$ given by (10) shows that it is constant, real and negative if the gain $A$ is greater than the unity.

$$
Z_{d s}=R_{d s}=R(1-A)
$$

As the current amplitude $a$ increases, the output voltage $u_{2}$ reaches the saturation level and becomes square shaped (Fig. 9.a) while the dipolar voltage $v_{d}$ becomes distorted (Fig. 9.b).
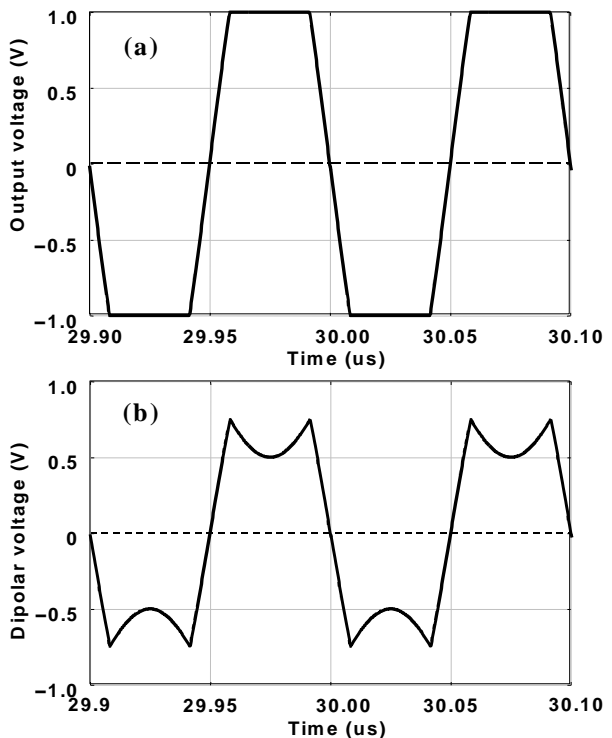

Fig. 9. Output and dipolar waveforms (hard saturation case).

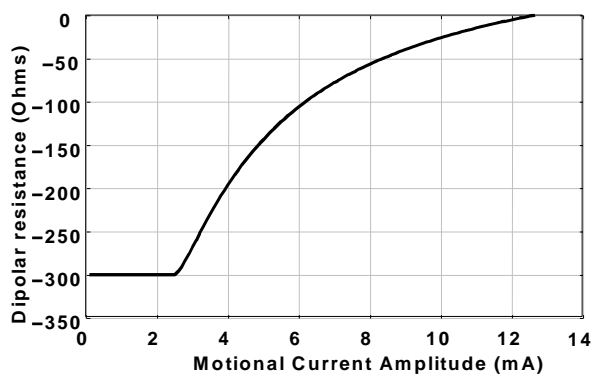

Fig. 10. Dipolar resistance (hard saturation case). 
By calculating the first harmonic of the dipolar voltage, it is possible to calculate the dipolar impedance as a function of the current amplitude $a$. In Fig. 10, it can be seen that the dipolar impedance $Z_{d}$ becomes nonlinear when the current amplitude is larger than the limit $a_{L}$ given by (11).

$$
a_{L}=\frac{V_{s a t}}{A R}
$$

In the present case, because there is no reactive part in the amplifier circuit, the dipolar impedance remains purely real so that $X_{d}=0$. Moreover, according to the expression of $R_{d s}$ given by (10), it is obvious that an inverting amplifier $(A<0)$ with real input and output impedances cannot be used in an oscillator circuit because $R_{d}$ is always positive.

\section{B. Cutoff Limiting}

Another limiting mechanism often involved in the oscillator circuits is the cutoff limitation an example of which is shown Fig. 11.

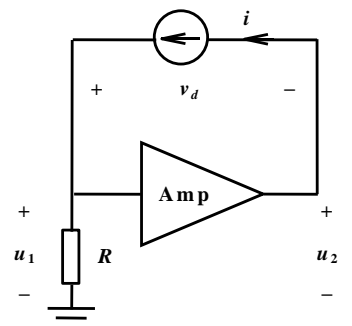

(a)

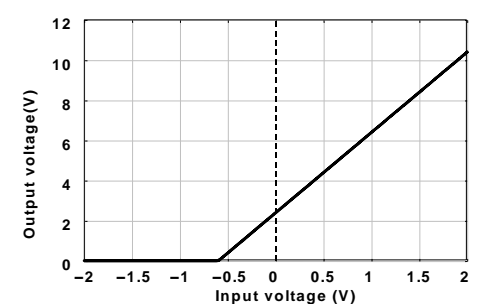

(b)
Fig. 11. Simple behavioral oscillator with cutoff limiting. Values used are: $A=4, R=100 \Omega, u_{0}=0.6 \mathrm{~V}$.

The oscillator shown has the same features as the previous ones except that the nonlinear transfer function is now given by the conditions (12) and has the shape represented Fig. 11.b.

$$
\begin{array}{lll}
u_{1} \leq-u_{0} & \Rightarrow & u_{2}=0 \\
u_{1} \geq-u_{0} & \Rightarrow & u_{2}=A\left(u_{1}+u_{0}\right)
\end{array}
$$
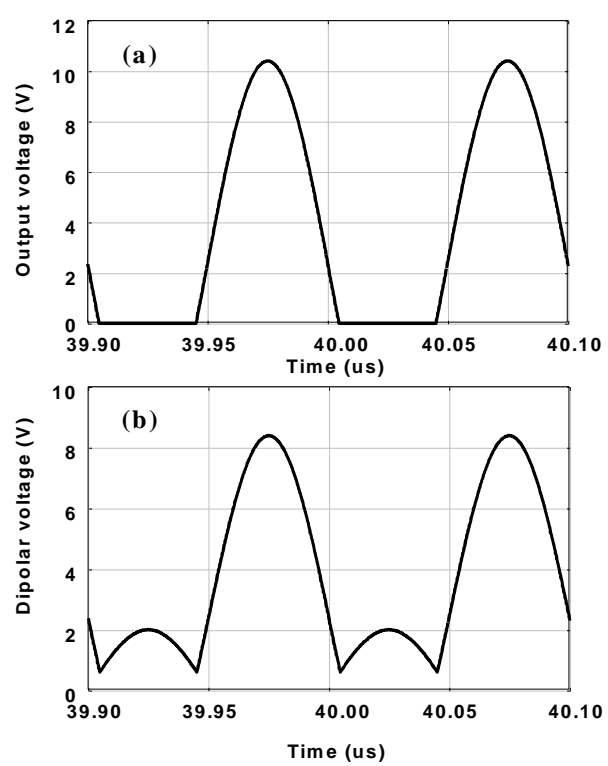

Fig. 12. Output and dipolar waveforms (cutoff case).
In this case, the output and dipolar voltages are no longer symmetrical (Fig. 12). As in the saturation case, for small current amplitude, the dipolar impedance is given by (10) as long as the cutoff amplitude is not reached. For increasing current amplitude, the dipolar impedance increases as shown in Fig. 13, while the dipolar reactance remains null if there is no reactive part in the amplifier.

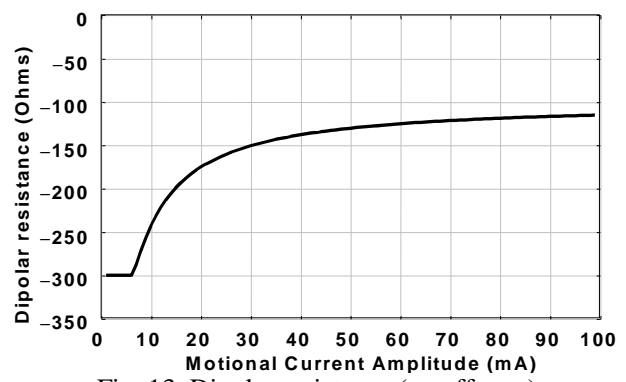

Fig. 13. Dipolar resistance (cutoff case).

\section{Soft Saturation Limiting}

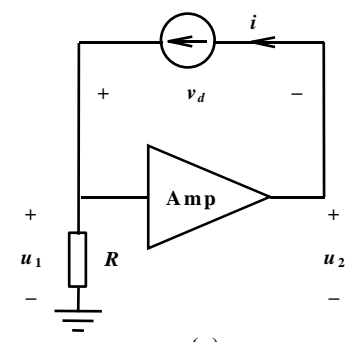

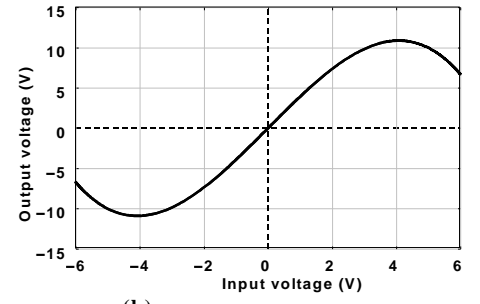

(b)
Fig. 14. Simple Van der Pol oscillator. Values used are: $A=4, R=100 \Omega, \varepsilon=210^{-2} \mathrm{~V}^{-2}$.
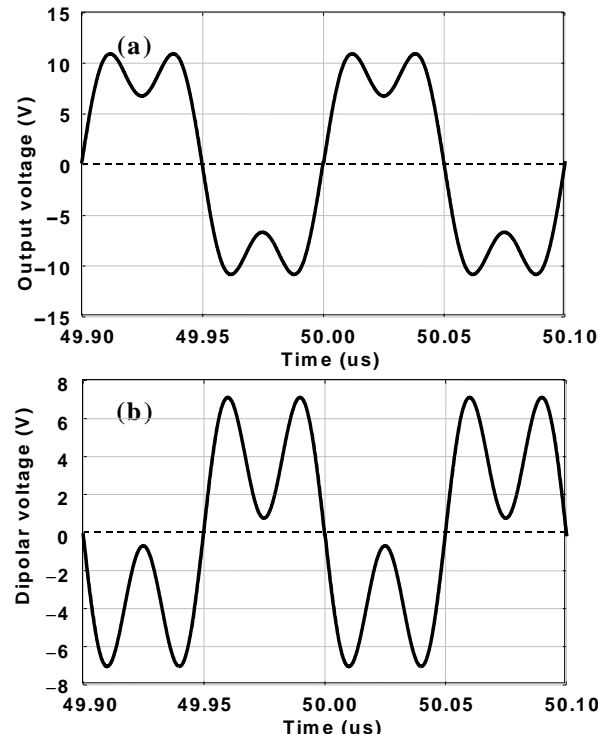

Fig. 15. Output and dipolar waveforms (soft saturation case).

Let us examine now the case of a soft limiting mechanism. A simple example of such a circuit is given by the Van der Pol oscillator that has the same representation as the previous ones, but in this case the limitation due to the nonlinear DC transfer function represented in Fig. 14.b is given by (13) where $A$ is the small signal gain and $\varepsilon$ is the nonli- 
near coefficient ( $A$ and $\varepsilon$ are assumed real and positive)

$$
u_{2}=A u_{1}\left(1-\varepsilon u_{1}^{2}\right)
$$

It is possible to show that in this case, the dipolar impedance can be derived under the form given by (14).

$$
Z_{d}=(1-A) R+\frac{3 A \varepsilon R^{3} a^{2}}{4}
$$

For small current amplitude, the dipolar impedance has the same expression (10) as in the two previous cases. As the current amplitude increases, the output and dipolar waveforms become distorted (Fig. 15), but, if there is no reactive part in the amplifier circuit, $Z_{d}$ remains real and increases with amplitude according to the parabolic law (14) (Fig. 16).

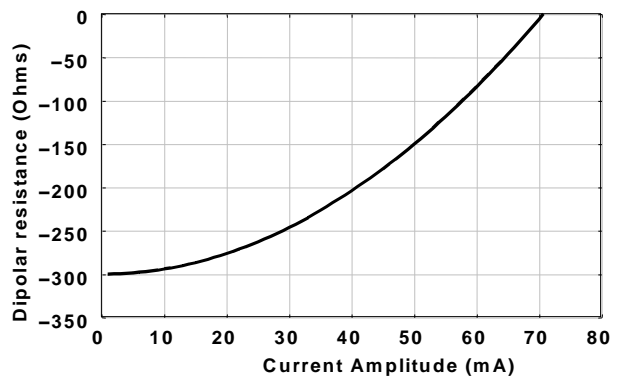

Fig. 16. Dipolar resistance (soft saturation case).

\section{FREQUENCY LIMITATION AND PARALlEL CAPACITANCE}

The simple models presented so far are only ideal behavioral models without any reactive component. Any actual amplifier has a reactive part at least because they have a limited bandwidth or because of the parallel capacitance that is included in the amplifier part as pointed out in section II.

\section{A. Amplifier Limited Bandwidth}
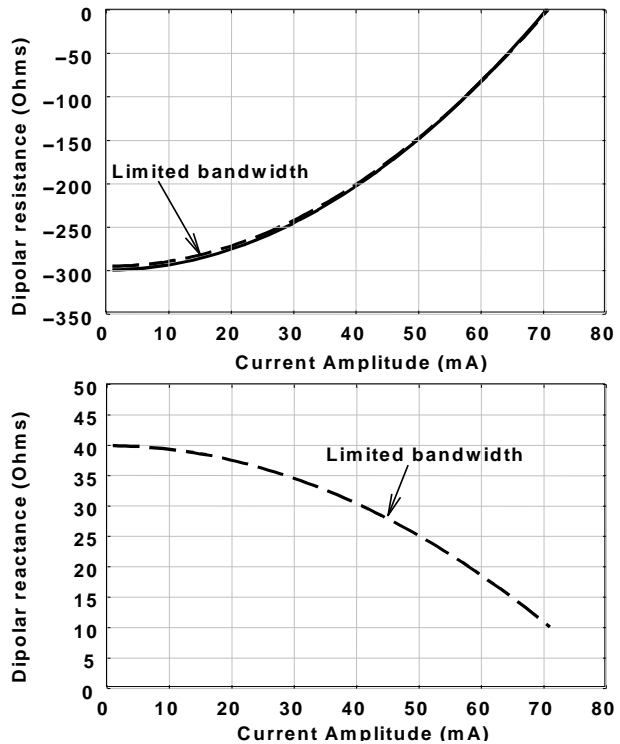

Fig. 17. Dipolar impedance of a limited bandwidth amplifier. Oscillation frequency: $10 \mathrm{MHz}$, cutoff frequency: $100 \mathrm{MHz}$.

Assuming that the linear part of the amplifier gain $A$ used in the previous cases in section IV has the form given by (15) where the cutoff frequency $\omega_{c}$ is much larger than the oscillation frequency, it is quite simple to demonstrate that in any case, the small signal dipolar impedance take the form (16) where it is obvious that the dipolar reactance is no longer null.

$$
\begin{aligned}
A= & \frac{A_{0}}{1+j \omega \tau_{c}} \quad \omega_{c}=\frac{1}{\tau_{c}}>\omega_{0} \\
& \left\{\begin{array}{l}
R_{d s}=\frac{R\left(1-A_{0}+\omega^{2} \tau_{c}^{2}\right)}{1+\omega^{2} \tau_{c}^{2}} \\
X_{d s}=\frac{R A_{0} \omega \tau_{c}}{1+\omega^{2} \tau_{c}^{2}}
\end{array}\right.
\end{aligned}
$$

When the amplifier has a limited bandwidth, it can be shown that the nonlinear part of the dipolar impedance does not depend strongly on the limiting mechanism, so it will be demonstrated only in the soft saturation case. Fig. 17 shows that the dipolar resistance remains practically unaffected while the dipolar reactance is now different from zero and decreases with the current amplitude.

\section{B. Parallel Capacitance}

As explained in section II, the parallel capacitance is included in the amplifier part of the oscillator circuit so that it can be considered in parallel with the dipolar impedance of the amplifier alone. Nevertheless, it is not so simple to express the equivalent dipolar impedance of these two components associated in parallel because the voltage across the dipole is no longer linear.
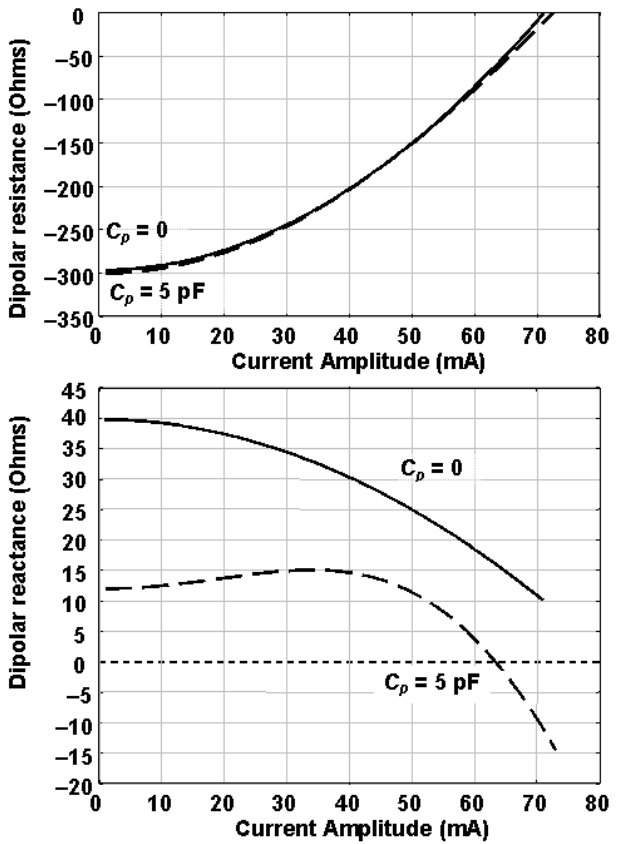

Fig. 18. Influence of the parallel capacitance on the dipolar impedance.

In Fig. 18 are compared the dipolar impedances of a limited bandwidth Van der Pol oscillator, as described in section IV $C$, with and without a parallel capacitance. As for the bandwidth effect, the dipolar resistance is practically not affected while the reactance exhibits a more important distortion. 


\section{INVERTING AMPLIFIER}

\section{A. Representation}

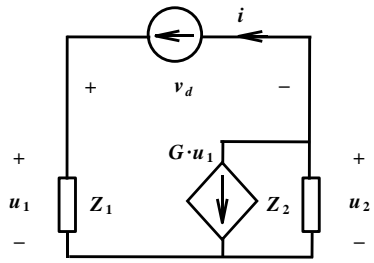

(a)

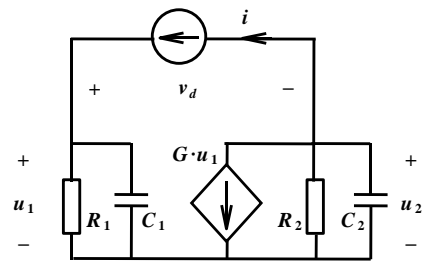

(b)
Fig. 19. Inverting amplifier.

All the amplifier circuits presented so far had a positive gain with real input and output impedances so that, even at low frequency, they may have a negative dipolar resistance. This is the reason why an oscillator using such an amplifier type is often called "negative resistance oscillator." Nevertheless, most of the current crystal oscillators are using an inverting amplifier that can be represented as in Fig. 19.a, the small signal dipolar impedance of which is given by (17) where the transconductance $G$ is real and positive.

$$
Z_{d s}=Z_{1}+Z_{2}+G Z_{1} Z_{2}=R_{d s}+j X_{d s}
$$

Obviously, this expression may have a negative real part only if the input and output impedances $Z_{1}$ and $Z_{2}$ both have an imaginary part. A simple form of such a case is represented in Fig. 19.b where $Z_{1}$ and $Z_{2}$ are parallel combinations of a resistance and a capacitance.

\section{B. Small Signal Analysis}

The small signal dipolar impedance (17) can be obtained by replacing $Z_{1}$ and $Z_{2}$ by their expression (18), thus the real part $R_{d s}$ and imaginary part $X_{d s}$ of the small signal dipolar impedance take the form (19) where $\tau_{1}$ and $\tau_{2}$ will be called the input and output time constant respectively.

$$
\begin{aligned}
\left\{\begin{array}{l}
Z_{1}=\frac{R_{1}}{1+\omega^{2} \tau_{1}^{2}} \quad \tau_{1}=R_{1} C_{1} \\
Z_{2}=\frac{R_{2}}{1+\omega^{2} \tau_{2}^{2}} \quad \tau_{2}=R_{2} C_{2}
\end{array}\right. \\
R_{d s}=\frac{R_{1}}{1+\omega^{2} \tau_{1}^{2}}+\frac{R_{2}}{1+\omega^{2} \tau_{2}^{2}}+\frac{G R_{1} R_{2}\left(1-\omega^{2} \tau_{1} \tau_{2}\right)}{\left(1+\omega^{2} \tau_{1}^{2}\right)\left(1+\omega^{2} \tau_{2}^{2}\right)} \\
X_{d s}=\frac{-R_{1} \omega \tau_{1}}{1+\omega^{2} \tau_{1}^{2}}-\frac{R_{2} \omega \tau_{2}}{1+\omega^{2} \tau_{2}^{2}}-\frac{G R_{1} R_{2}\left(\omega \tau_{1}+\omega \tau_{2}\right)}{\left(1+\omega^{2} \tau_{1}^{2}\right)\left(1+\omega^{2} \tau_{2}^{2}\right)} \\
G^{2}>G_{c}=\frac{C_{1}}{\tau_{2}}+\frac{C_{2}}{\tau_{1}} \\
\omega^{2}>\omega_{c}^{2}=\frac{1}{\tau_{1} \tau_{2}} \frac{G-\left(\frac{1}{R_{1}}+\frac{1}{R_{2}}\right.}{G}
\end{aligned}
$$

By looking at the expression of $R_{d s}$ it should be demonstrated that it can be negative only if two conditions are ful- filled: the tranconductance $G$ must be larger than the limit $G_{c}$ given by (20) and the frequency must be higher than the limit $\omega_{c}$ given by (21).

The small signal dipolar impedance for a given pair of impedances $Z_{1}$ and $Z_{2}$ is represented in Fig. 20 where oscillation cannot start if $R_{d s}$ is in the shaded area. Because $Z_{1}$ and $Z_{2}$ play symmetrical roles in the expression of the small signal dipolar impedance, the same curves are obtained when they are reversed.

Unlike the case of the non inverting amplifier with real input and output impedances, here the parallel capacitance strongly modify both the real and imaginary parts of the small signal dipolar impedance as shown in Fig. 20 where it can also be seen that the critical frequency $\omega_{c}$ below which the oscillation cannot start, is not affected by the parallel capacitance. In addition, as for the case where the parallel capacitance is null, the same small signal dipolar impedance is obtained when the input and output impedances are reversed. It should be emphasized that the results presented so far in this section do not depend on the limiting mechanism but only on the small signal transconductance as well as the input and output impedance values.
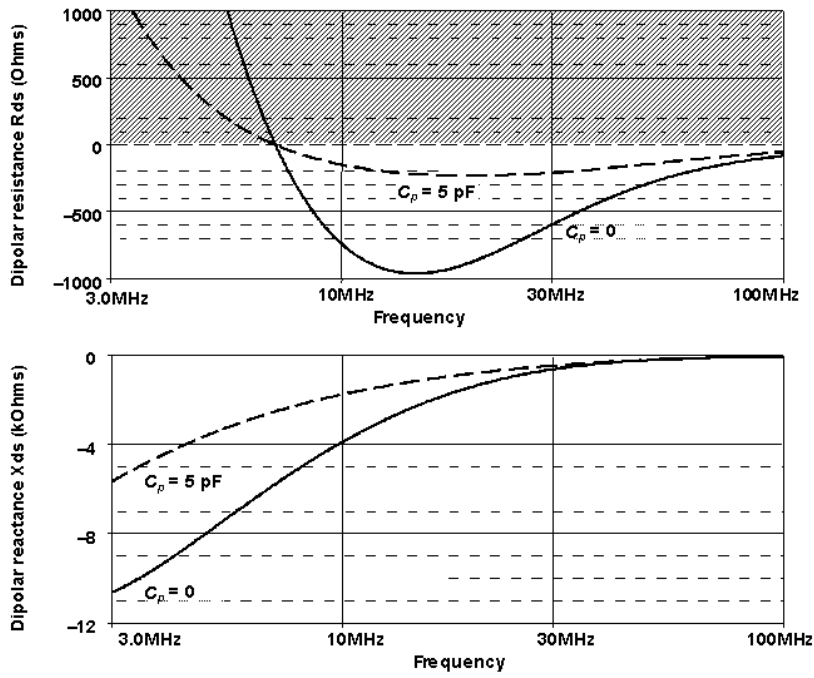

Fig. 20. Small signal dipolar impedance of an inverting amplifier. $G=100 \mathrm{~mA} / \mathrm{V}, C_{1}=C_{2}=75 \mathrm{pF}, R_{1}=100 \Omega, R_{2}=1000 \Omega$.

\section{Transconductance Amplifier with Cutoff Limiting}

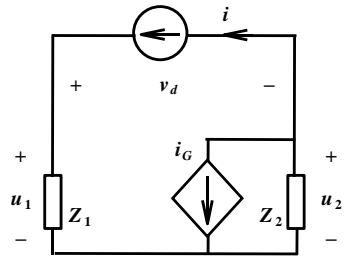

(a)

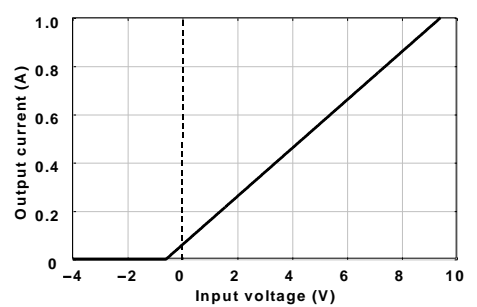

(b)
Fig. 21. Transconductance amplifier with cutoff limiting. $G=100 \mathrm{~mA} / \mathrm{V}, u_{0}=0.6 \mathrm{~V}$.

Several simulations performed on transconductance amplifiers with different limitation mechanisms have shown that the input and output impedances (or time constants) 
have similar effects on the nonlinear dipolar impedance. Thus, the attention will be focused only on the cutoff limiting mechanism often involved in the oscillator circuits. In this case, the nonlinear transconductance represented in Fig. 21.b is defined by the conditions (22).

$$
\begin{array}{lll}
u_{1} \leq-u_{0} & \Rightarrow & i_{G}=0 \\
u_{1} \geq-u_{0} & \Rightarrow & i_{G}=G\left(u_{1}+u_{0}\right)
\end{array}
$$
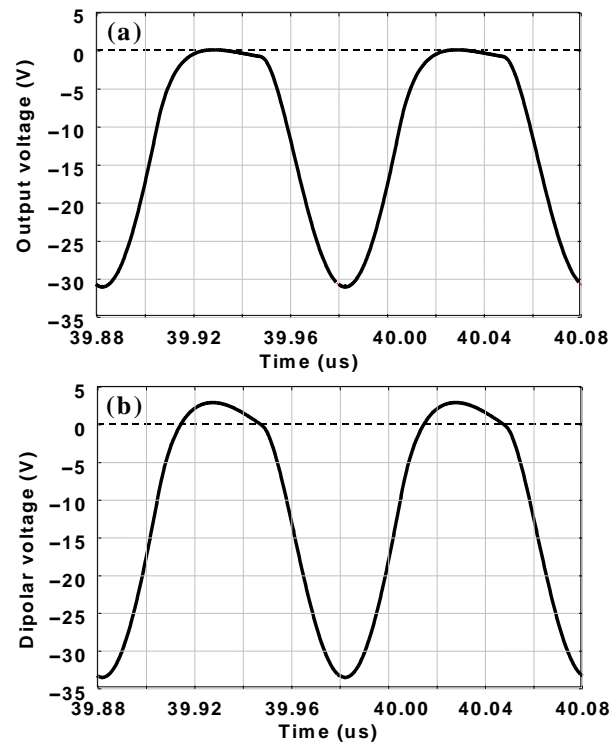

Fig. 22. Output and dipolar waveforms (transconductance amplifier with cutoff limiting).
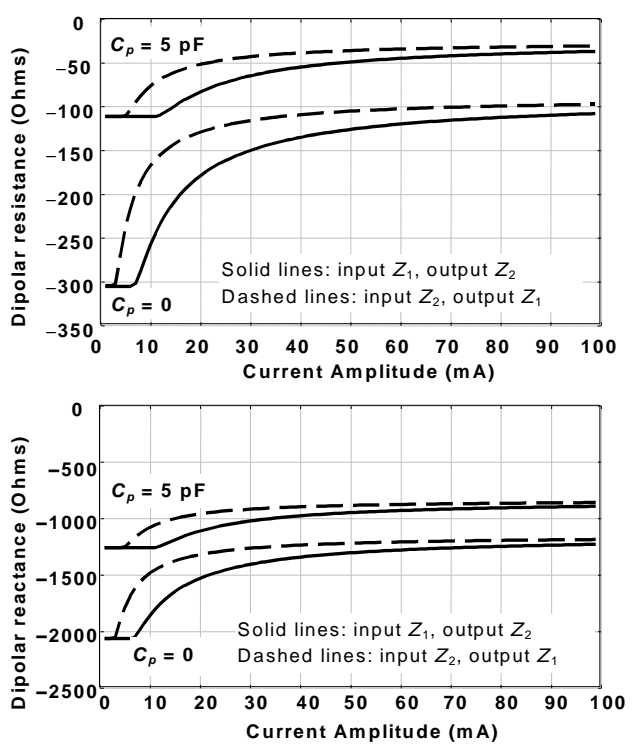

Fig. 23. Dipolar impedance of a transconductance amplifier. $Z_{1}: R_{1}=100 \Omega / / C_{1}=75 \mathrm{pF}, Z_{2}: R_{2}=1000 \Omega / / C_{2}=75 \mathrm{pF}$.

The output and dipolar waveforms of the transconductance amplifier with cutoff limiting for a given pair of impedances $Z_{1}$ and $Z_{2}$ are shown in Fig. 22, and its dipolar impedance is represented in Fig. 23. Note that the dipolar resistance looks like the one of a non inverting amplifier having the same limiting mechanism (Fig. 13) but with a much larger reactance value. As demonstrated in section VI $B$, when the two impedances $Z_{1}$ and $Z_{2}$ are reversed, the dipolar resistance and reactance keep the same value for small current amplitude but, as the amplitude increases, the two curves may have a quite different location. As for the small dipolar impedance, the parallel capacitance strongly modify both the dipolar resistance and reactance of the amplifier (Fig. 23). By looking at (6) it is obvious that a negative reactance corresponds to a positive frequency shift that can become very large if the resonator motional inductance $L_{q}$ is not much greater than the dipolar inductance $L_{d}$. In such a case, the oscillation frequency may be close to the resonator antiresonant frequency, this is the reason why these oscillators are often improperly called "parallel resonance oscillators."

\section{Input and Output Impedances}
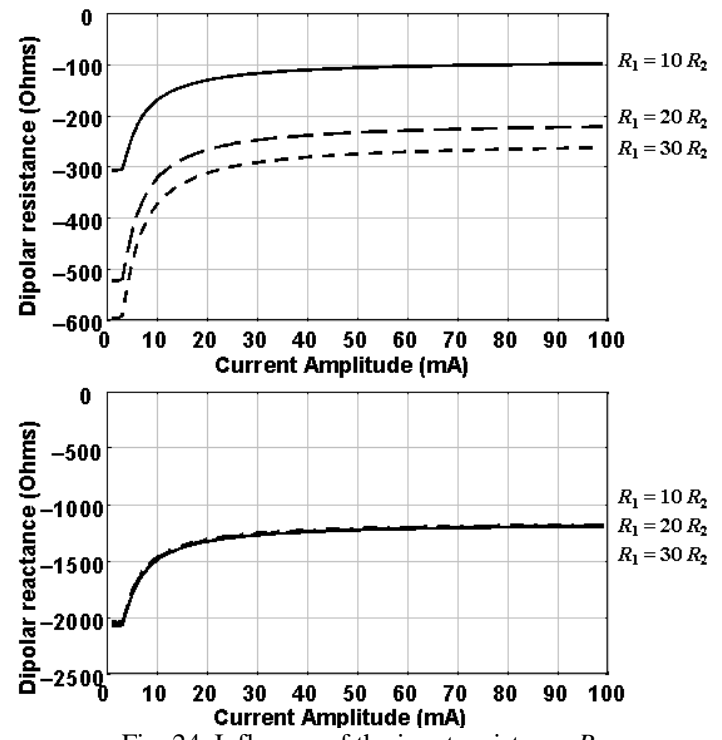

Fig. 24. Influence of the input resistance $R_{1}$.

$C_{1}=C_{2}=75 \mathrm{pF}, R_{2}=100 \Omega$.
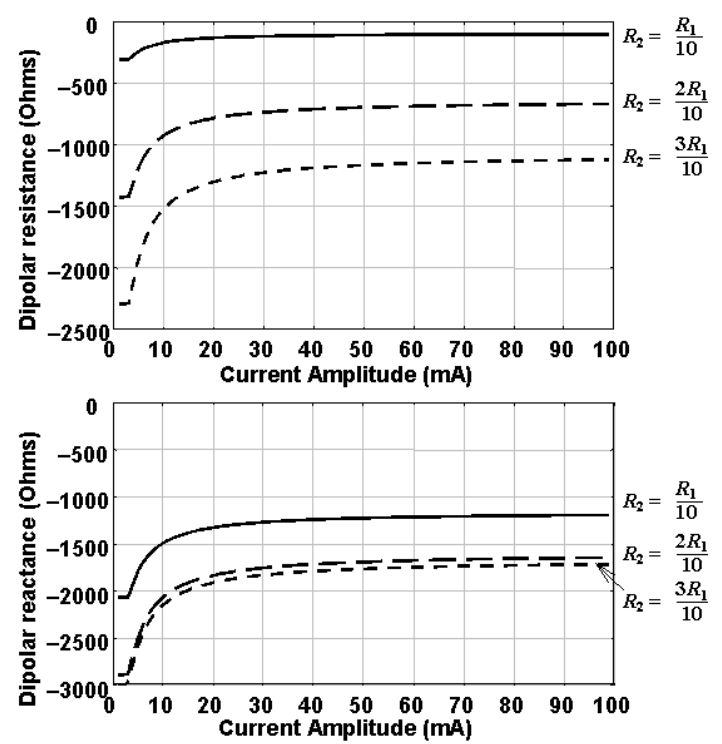

Fig. 25. Influence of the output resistance $R_{2}$. $C_{1}=C_{2}=75 \mathrm{pF}, R_{1}=1000 \Omega$. 

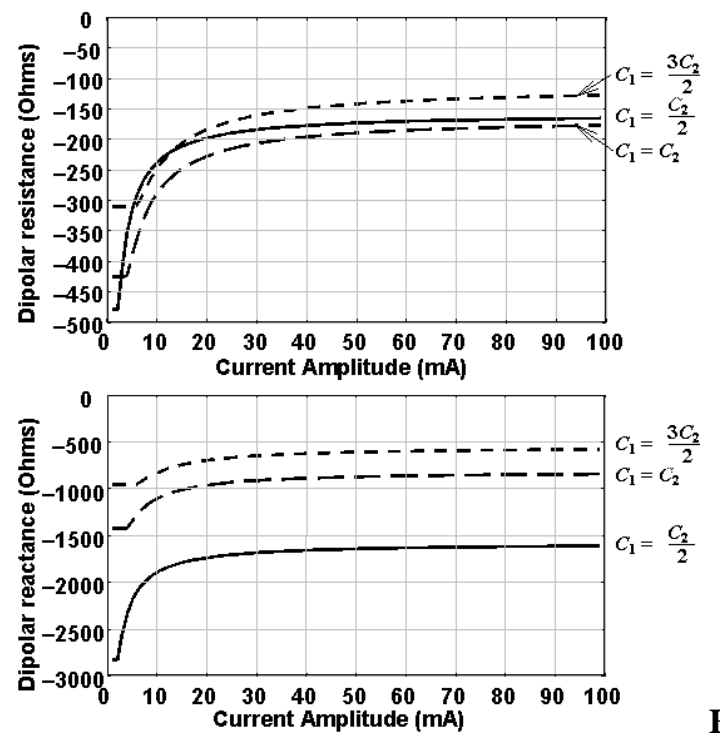

Liaison incorrecte.

Fig. 26. Influence of the input capacitance $C_{1}$. $R_{1}=1000 \Omega, R_{2}=100 \Omega, C_{2}=100 \mathrm{pF}$.

Figures 24 to 27 show how the dipolar impedance of the transconductance amplifier previously used is modified when one of the four components defining the input and output impedances $Z_{1}$ and $Z_{2}$ is modified, the three others being kept constant. Of course, these figures do not cover all the possible combinations but only demonstrate how it is possible to use the dipolar method to optimize a circuit. It is obvious in Figs. 24 and 25, for example, that modifying the input or output resistance $R_{1}$ or $R_{2}$ drastically changes the dipolar resistance and therefore the motional current amplitude in the crystal, while the frequency shift due to the dipolar reactance is more sensitive to a change in the output resistance than in the input resistance.

On the other hand, the dipolar resistance appears more sensitive to a change in the output capacitance than in the input capacitance, while both have an important effect in the dipolar reactance as shown in Figs. 26 and 27.

\section{CONCLUSION}

In this work, the most important amplifier parameters that play a part in the behavior of quartz crystal oscillators have been studied by using the nonlinear dipolar that is a powerful and well suited method to analyze the characteristics and performance of high Q-factor circuits. The dipolar analysis has been successfully implemented in a dedicated software (ADOQ) whose efficiency and accuracy have been checked experimentally [10]. In addition to the main features described in the introduction, the program is currently being completed by a optimization module intended to help the designer in choosing the right circuit for a given purpose or to choose the right components for a given design.
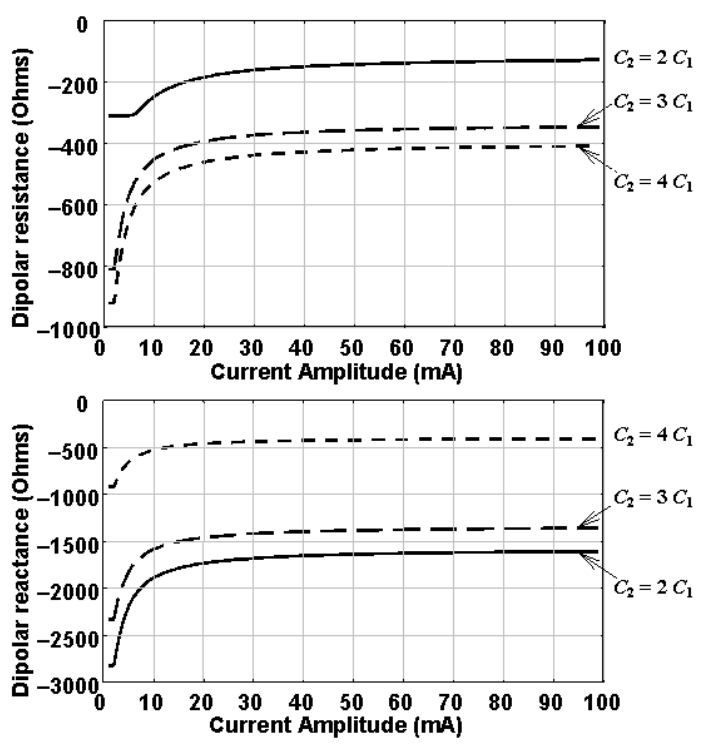

Fig. 27. Influence of the output capacitance $C_{2}$. $R_{1}=1000 \Omega, R_{2}=100 \Omega, C_{1}=50 \mathrm{pF}$.

\section{REFERENCES}

[1] R. Brendel, D. Gillet, N. Ratier, F. Lardet-Vieudrin, and J. Delporte, "Nonlinear dipolar modeling of quartz oscillators," Proc. of the $14^{\text {th }}$ EFTF, Torino, Italy, pp. 184-188, March 2000.

[2] M. Addouche, R. Brendel, D. Gillet, N. Ratier, F. Lardet-Vieudrin, and J. Delporte, "Modeling of quartz crystal oscillators by using nonlinear dipolar method," IEEE Trans. UFFC, vol. 50, pp. 487-495, May 2003.

[3] M. Addouche, N. Ratier, D. Gillet, R. Brendel, F. Lardet-Vieudrin, and J. Delporte, "ADOQ: a quartz crystal oscillator simulation software," Proc. of the 55 th IEEE IFCS, Seattle, USA, pp. 753-757, June 2001.

[4] K. Kurokawa, "Some basic characteristics of broadband negative resistance oscillator circuits," Bell System Technical Journal, pp. 1937-1955, July-August 1969.

[5] E.A. Vittoz, "Quartz oscillators for watches," Proc. of the $10^{\text {th }}$ International Congress of Chronometry, pp. 131-140, Geneva, Switzerland, September 1979.

[6] N. Kryloff, and N.N. Bogoliuboff, Introduction to Nonlinear Mechanics, Princeton University Press, 1943.

[7] A.A. Andronov, A.A. Vitt, and S.E. Khaikin, Theory of Oscillators, New York, Pergamon Press, 1966, pp. 585-613.

[8] R. Brendel, D. Gillet, N. Ratier, M. Addouche, and J. Delporte, "Oscillator noise simulation by using nonlinear dipolar method," Proc. of the 15 $5^{\text {th }}$ EFTF, Neuchâtel, Switzerland, pp. 123-128, March 2001.

[9] B. Parzen, A. Ballato, Design of Crystal and other Harmonic Oscillators, New York, Wiley Intersciences, 1983.

[10] M. Addouche, N. Ratier, D. Gillet, R. Brendel, and J. Delporte, "Experimental validation of the nonlinear dipolar method," Proc. of the $16^{\text {th }}$ EFTF, St. Petersburg, Russia, March 2002. 